\title{
Critical Combinations of Higher-Order Terms in Einstein-Maxwell Theory and Compactification
}

\author{
Nahomi Kan ${ }^{1}$ and Kiyoshi Shiraishi ${ }^{2}$ \\ ${ }^{1}$ National Institute of Technology, Gifu College, Motosu-shi, Gifu 501-0495, Japan \\ ${ }^{2}$ Graduate School of Science and Engineering, Yamaguchi University, Yamaguchi-shi, Yamaguchi 753-8512, Japan \\ Correspondence should be addressed to Kiyoshi Shiraishi; shiraish@yamaguchi-u.ac.jp
}

Received 27 August 2015; Accepted 1 November 2015

Academic Editor: Chao-Qiang Geng

Copyright (c) 2015 N. Kan and K. Shiraishi. This is an open access article distributed under the Creative Commons Attribution License, which permits unrestricted use, distribution, and reproduction in any medium, provided the original work is properly cited. The publication of this article was funded by SCOAP ${ }^{3}$.

\begin{abstract}
We discuss the role of a particular combination of higher derivative terms in higher dimensional theories, particularly in the background of spontaneous compactification. Two classes of theories are proposed in this paper. The first model as a generalization of the critical gravity with the Maxwell field could have a de Sitter solution. We consider the Lanczos-Lovelock term and Horndeski term as well as the higher-order Maxwell term for the second model, which contains a possible longer expansion time for the inflationary phase. It is interesting that both models can be regarded as the generalization of the Randjbar-Daemi, Salam and Strathdee (RSS) model and give the well behavior for inflation stage under the specific assumptions.
\end{abstract}

\section{Introduction}

Over several years, the gravitational theory with higher derivative terms $[1,2]$ has been a highly interesting subject studied in physics. The treatment of Einstein gravity with perturbative field quantization naturally leads to the inclusion of such nonlinear terms in the spacetime curvatures for effective actions.

The generalization of Einstein gravity has been developed extensively by many authors. The Lanczos-Lovelock gravity [3-6] (for a review, see [7]), which is dictated by effective action with the dimensionally continued Euler forms, is defined in a generic dimensional spacetime, and it describes a massless spin-two fluctuation despite the existence of higherorder terms in its action. About a few decades ago, it was discovered that the effective field theory arising from string theory involves similar terms at least for the lowest-order correction to the Einstein-Hilbert term $[8,9]$.

The other specific extension is found in the study on the so-called critical gravity [10-12] (although the word "critical" originally implied the emergence of Log gravity [13-15]). In this gravitational theory, the behavior of spin-two modes is described by the Lagrangian of a special combination of higher-order terms in the Ricci tensor and the scalar curvature. There is a ghost field but no spin-zero mode in this theory. In the three-dimensional spacetime, the model governed by a similar Lagrangian expresses the new massive gravity $[16,17]$.

In this study, we consider incorporation of the Maxwell fields to the (next-to) critical gravity, which has been studied under the assumption of the maximally symmetric background spacetime. The higher derivative theory for the other type of background fields is worth studying. Inclusion of a nonvanishing flux field brings about partial compactification with a maximally symmetric extra space and might provide an interesting scenario for a very early stage of the universe.

The assumption of the partially symmetric solution could yield another possibility. It is feasible to attach the other type of higher-order terms to the Lagrangian which realizes the spontaneous compactification. Due to the symmetry, the use of the Riemann tensor is possible in the additional terms, which are expressed by special combinations of GaussBonnet terms and Horndeski's generalized Maxwell term [18]. These terms induce a second-order field equation displaying the cosmological evolution of the background geometry (the applications of the Horndeski's vector-tensor 
theory have been discussed previously in four-dimensional $[19,20]$ and in six-dimensional cosmology [21]).

This paper is organized as follows. In Section 2, we build the Lagrangian of our first model, which involves the quadratic terms of the Ricci tensor, the Ricci scalar, and the two-form flux field, in a similar way as revealed before in the study of critical gravity. The spontaneous compactification in the first model is studied in Section 3 and cosmological solutions are examined in Section 4. In Section 5, we present the second model containing the quadratic term of the Riemann tensor. We also investigate the cosmological solution qualitatively in this model. The last section is devoted to the summary and further research prospects in this area of study.

It is to be noted that throughout this study, we deal with our models at the classical level only.

\section{A Critical Modification of Einstein-Maxwell Theory}

In the study on critical gravity, it is found that the Lagrangian of the specific combination of curvatures yields a spintwo massless graviton and a ghost mode; there appears no scalar mode as in Einstein gravity. In this section, we propose a model of higher-order extension of the MaxwellEinstein theory. We will follow the method for constructing the Lagrangian examined in our previous study [22, 23]. In $[22,23]$, we constructed the action of higher derivative pure gravity which admits the (anti-)de Sitter solution. In similar sense, we manage to construct the model with higherorder terms in which background fields satisfy the Einstein equation in the presence of the Maxwell field in this paper.

First, we start with the action for the Einstein gravity and electromagnetism in $D$ dimensions:

$$
I_{0}=\int d^{D} x \sqrt{-g} L_{0}=\int d^{D} x \sqrt{-g}\left[R-F_{M N} F^{M N}-\Lambda\right],
$$

where $R$ and $\Lambda$ denote the scalar curvature obtained from the metric $g_{M N}$ and the cosmological constant, respectively. The field strength $F_{M N}$ is defined by $F_{M N} \equiv \partial_{M} A_{N}-\partial_{N} A_{M}$ with a vector field $A_{M}$ and $F^{2} \equiv F_{M N} F^{M N}$. The suffixes $M, N, \ldots$ vary over $0,1, \ldots, D-1$.

Apply the variational principle to the action results in the following equations of motion:

$$
T_{M N}=0
$$

with

$$
\begin{aligned}
T_{M N} \equiv & \frac{1}{\sqrt{-g}} \frac{\delta I_{0}}{\delta g^{M N}} \\
= & R_{M N}-\frac{1}{2} R g_{M N}-2\left(F_{M N}^{2}-\frac{1}{4} F^{2} g_{M N}\right) \\
& +\frac{1}{2} \Lambda g_{M N},
\end{aligned}
$$

where $F_{M N}^{2} \equiv F_{M P} F_{N}{ }^{P}$ and

$$
J^{N} \equiv \nabla_{M} F^{M N}=0 .
$$

Next, we construct a Lagrangian for our model as follows:

$$
L=\alpha L_{0}-\beta T^{M N} \Delta_{M N P Q} T^{P Q},
$$

where

$$
\Delta_{M N P Q} \equiv \frac{1}{2}\left(g_{M P} g_{N Q}+g_{M Q} g_{N P}\right)-\frac{1}{D-1} g_{M N} g_{P Q}
$$

Note that the solution of $T_{M N}=0$ is the solution for the equation of motion derived from $L$, provided that the field equation for the vector field is satisfied (though the stability is not necessarily guaranteed). (Generally speaking, we can add the term $\propto J_{M} J^{M}$ to our Lagrangian, but we restrict ourselves to the case without such a term in the present paper.)

The Lagrangian $L$ can then be written as

$$
\begin{aligned}
L & =\alpha\left[R-F^{2}-\Lambda\right]-\beta\left[R_{M N} R^{M N}-\frac{D}{4(D-1)} R^{2}\right. \\
& -4 F_{M N}^{2} R^{M N}+\frac{D+2}{2(D-1)} F^{2} R+4 F_{M N}^{2} F^{2^{M N}} \\
& -\frac{D+8}{4(D-1)}\left(F^{2}\right)^{2} \\
& \left.+\frac{\Lambda}{2(D-1)}\left\{(D-2) R-(D-4) F^{2}-\frac{D}{2} \Lambda\right\}\right] .
\end{aligned}
$$

Incidentally, we can pick up some "critical" cases: if $\alpha=(D / 4(D-1)) \beta \Lambda$, the contribution of the cosmological constant is zero in the $D$-dimensional spacetime; if $\alpha=((D-$ 2) $/ 2(D-1)) \beta \Lambda$, the Einstein-Hilbert term $(R)$ is absent from the $D$-dimensional action; and if $\alpha=((D-4) / 2(D-1)) \beta \Lambda$, the pure Maxwell term $\left(F^{2}\right)$ is absent from the $D$-dimensional action.

We should note that the auxiliary field method $[14,16,17$, 23], which can be used in critical higher-order gravity, also leads to the additional terms such as

$$
L^{\prime}=\alpha L_{0}-\beta\left(2 T_{M N} S^{M N}-S_{M N} S^{M N}+S^{2}\right),
$$

where $S^{M N}$ is an auxiliary symmetric tensor field and $S \equiv$ $S^{M N} g_{M N}$. Varying the auxiliary field $S^{M N}$, we obtain

$$
T_{M N}=S_{M N}-S g_{M N},
$$

or, solving this equation with respect to $S_{M N}$, we get

$$
S_{M N}=T_{M N}-\frac{1}{D-1} T g_{M N}=\Delta_{M N P Q} T^{P Q} .
$$

Therefore, one can see the equivalence between the Lagrangians, $L$ and $L^{\prime}$. We can derive the linearized field equation using the action $I^{\prime}=\int d^{D} x L^{\prime}$.

Now, we investigate the behavior of the linear fluctuations around the background geometry, which satisfies $T^{M N}=0$. We decompose the metric as follows:

$$
g_{M N}=\bar{g}_{M N}+h_{M N}
$$


The indices are raised and lowered by the background metric, $\bar{g}_{M N}$. Then, the trace of the fluctuation is expressed as

$$
h \equiv \bar{g}^{M N} h_{M N} .
$$

For convenience, we write down the curvature tensors up to the linear order in $h_{M N}$ here as [24]

$$
\begin{aligned}
& R_{P Q}^{M N}=\bar{R}_{P Q}^{M N}-\frac{1}{2} \bar{R}_{P Q}^{M L} h_{L}^{N}-\frac{1}{2} \bar{R}_{P Q}^{N L} h_{L}^{M} \\
& +\frac{1}{2}\left(-\bar{\nabla}_{P} \bar{\nabla}^{M} h_{\mathrm{Q}}^{N}+\bar{\nabla}_{P} \bar{\nabla}^{N} h_{\mathrm{Q}}^{M}+\bar{\nabla}_{\mathrm{Q}} \bar{\nabla}^{M} h_{P}^{N}\right. \\
& \left.-\bar{\nabla}_{Q} \bar{\nabla}^{N} h_{P}^{M}\right),
\end{aligned}
$$

$$
\begin{aligned}
R_{N}^{M} & =\bar{R}_{N}^{M}-\bar{R}_{N Q}^{M P} h_{P}^{Q}+\frac{1}{2} \bar{R}_{L}^{M} h_{N}^{L}-\frac{1}{2} \bar{R}_{N}^{L} h_{L}^{M} \\
& +\frac{1}{2}\left(-\bar{\nabla}^{2} h_{N}^{M}-\bar{\nabla}_{N} \bar{\nabla}^{M} h+\bar{\nabla}^{M} \bar{\nabla}_{L} h_{N}^{L}+\bar{\nabla}_{N} \bar{\nabla}_{L} h^{L M}\right), \\
R & =\bar{R}-\bar{R}^{M N} h_{M N}+\bar{\nabla}_{M} \bar{\nabla}_{N} h^{M N}-\bar{\nabla}^{2} h,
\end{aligned}
$$

where the nabla stands for the covariant derivative and the barred symbols show that they are constructed from the background metric, $\bar{g}_{M N}$.

Assuming the background field satisfies $\bar{T}_{M N}=\bar{R}_{M N}-$ $(1 / 2) \bar{R} \bar{g}_{M N}-2\left(\bar{F}_{M N}^{2}-(1 / 4) \bar{F}^{2} \bar{g}_{M N}\right)+(1 / 2) \Lambda \bar{g}_{M N}=0$, we can use

$$
\delta\left(2 S^{P Q} T_{P Q}-S_{P Q} S^{P Q}+S^{2}\right)=2 S^{P Q} \delta T_{P Q}+\text { higher-order terms in small fluctuations from the background, }
$$

to obtain the field equation with a linearized order:

$$
\begin{aligned}
& \frac{1}{\sqrt{-g}} \frac{\delta I^{\prime}}{\delta g_{M N}}=0 \longrightarrow \\
& -\alpha T^{M N}-\beta\left[-\bar{\nabla}^{2} S^{M N}-\bar{\nabla}^{M} \bar{\nabla}^{N} S+\bar{\nabla}^{M} \bar{\nabla}_{L} S^{L N}\right. \\
& \quad+\bar{\nabla}^{N} \bar{\nabla}_{L} S^{L M}-\bar{\nabla}_{P} \bar{\nabla}_{Q} S^{P Q} \bar{g}^{M N}+\bar{\nabla}^{2} S \bar{g}^{M N} \\
& \quad-2 \bar{R}_{P}{ }^{M}{ }^{N} S^{P Q}+\bar{R}_{P Q} S^{P Q} \bar{g}^{M N}-\bar{R} S^{M N}+\bar{R}_{L}^{M} S^{L N} \\
& +\bar{R}_{L}^{N} S^{L M}+\Lambda S^{M N}+4 \bar{F}^{M}{ }_{P}{ }^{N}{ }_{Q} S^{P Q}+\bar{F}^{2} S^{M N} \\
& \left.-2 \bar{F}_{P Q}^{2} S^{P Q} \bar{g}^{M N}\right]=0 .
\end{aligned}
$$

Noting that $S^{M N}$ is in the linear order $\left(\bar{S}^{M N}=0\right)$, we have used the condition $\bar{T}_{M N}=0$ for the background field.

The generalized Maxwell equation in the linear order of fluctuations takes the form

$$
\begin{aligned}
& \frac{1}{\sqrt{-g}} \frac{\delta I^{\prime}}{\delta A_{N}}=0 \longrightarrow \\
& 2 \alpha \bar{\nabla}_{M} f^{M N}-2 \beta \bar{\nabla}_{M}\left[2 \bar{F}^{M}{ }_{P} S^{P N}-2 \bar{F}_{P}^{N} S^{P M}-\bar{F}^{M N} S\right] \\
& \quad=0
\end{aligned}
$$

where $f_{M N}=F_{M N}-\bar{F}_{M N}$.

The linearized equation obtained from (9) is

$$
\begin{aligned}
& T^{M N}=S^{M N}-g^{M N} S \longrightarrow \\
& \frac{1}{2}\left[-\bar{\nabla}^{2} h^{M N}-\bar{\nabla}^{M} \bar{\nabla}^{N} h+\bar{\nabla}^{M} \bar{\nabla}_{L} h^{L N}+\bar{\nabla}^{N} \bar{\nabla}_{L} h^{L M}\right.
\end{aligned}
$$

$$
\begin{aligned}
& -\bar{\nabla}_{P} \bar{\nabla}_{\mathrm{Q}} h^{P Q} \bar{g}^{M N}+\bar{\nabla}^{2} h \bar{g}^{M N}-2 \bar{R}_{P}{ }^{M}{ }_{\mathrm{Q}}{ }^{N} h^{P Q} \\
& +\bar{R}^{P Q} h_{P Q} \bar{g}^{M N}-\bar{R} h^{M N}+\bar{R}_{L}^{M} h^{L N}+\bar{R}_{L}^{N} h^{L M} \\
& \left.+\Lambda h^{M N}\right]+2 \bar{F}_{P}^{M} \bar{F}^{N}{ }_{Q} h^{P Q}+\frac{1}{2} \bar{F}^{2} h^{M N} \\
& -\bar{F}_{P Q}^{2} h^{P Q} \bar{g}^{M N}-2 \bar{F}^{P M} f_{P}{ }^{N}-2 \bar{F}^{P N} f_{P}{ }^{M} \\
& +\bar{F}^{P Q} f_{P Q} \bar{g}^{M N}=S^{M N}-\bar{g}^{M N} S .
\end{aligned}
$$

The important observation is that, using (9), the field equation (15) becomes

$$
\begin{aligned}
-\bar{\nabla}^{2} S^{M N}-\bar{\nabla}^{M} \bar{\nabla}^{N} S+\bar{\nabla}^{M} \bar{\nabla}^{L} S^{L N}+\bar{\nabla}^{N} \bar{\nabla}^{L} S^{L M} \\
-\bar{\nabla}_{P} \bar{\nabla}_{Q} S^{P Q} \bar{g}^{M N}+\bar{\nabla}^{2} S \bar{g}^{M N} \\
+\frac{\alpha}{\beta}\left(S^{M N}-\bar{g}^{M N} S\right)-2 \bar{R}_{P}^{M}{ }_{Q}^{N} S^{P Q} \\
+\bar{R}_{P Q} S^{P Q} \bar{g}^{M N}-\bar{R} S^{M N}+\bar{R}_{L}^{M} S^{L N}+\bar{R}_{L}^{N} S^{L M} \\
+\Lambda S^{M N}+4 \bar{F}^{M}{ }_{P} \bar{F}^{N} S^{P Q}+\bar{F}^{2} S^{M N} \\
+2 \bar{F}_{P Q}^{2} S^{P Q} \bar{g}^{M N}=0 .
\end{aligned}
$$

In the $D$-dimensional Minkowski vacuum, this equation is just the Fierz-Pauli equation [25] for the spin-two wave with mass-squared $m^{2}=\alpha / \beta$. Therefore, we recognize that $S^{M N}$ corresponds to the massive ghost field. The postulation of no tachyonic ghost requires $\alpha / \beta>0$ in the present model.

The linear fluctuation from the background fields can be investigated by using the above equations. In the next section, we consider the solution for spontaneous compactification of spacetime as the background. 


\section{Spontaneous Compactification in Critically Modified Higher-Order Einstein-Maxwell Theory}

In this section, we study the partial compactification of space with a nontrivial flux in our model. The extra dimensions are considered to be compactified into a sufficiently small size. A partially symmetric solution in our model describes compactification of the form $M_{D-2} \otimes S^{2}$, where $M_{D-2}$ is the $(D-2)$ dimensional Minkowski spacetime and $S^{2}$ is a twodimensional sphere, as in the work of Randjbar-Daemi, Salam and Strathdee (RSS) [26], where $D=6$ is considered. We consider the solution for the flat $D-2$ dimensional spacetime and study its classical stability in this section, though other symmetric spacetimes are also interesting. Our notation is that the suffixes $\mu, \nu, \ldots$ run over $0,1,2, D-3$ and the suffixes $m, n, \ldots$ are used for the extra dimensions of $D-2$ and $D-1$.

As stated in the previous section, the solution for $T_{M N}=0$ and $J_{M}=0$ is a solution in our model. A suitable ansatz for the flux field is

$$
F=\frac{1}{2} F_{M N} d x^{M} \wedge d x^{N}=\frac{1}{2} q \varepsilon_{m n} d x^{m} \wedge d x^{n}
$$

where $\varepsilon_{m n}$ is the antisymmetric symbol and the constant $q$ is the strength of the two-form flux. (It can be checked that this configuration is the solution of our model.) This monopole flux yields the relation,

$$
F_{m n}^{2}=\frac{1}{2} F^{2} g_{m n}
$$

Then, the equation, $T_{M N}=0$, can be decomposed to

$$
\begin{aligned}
R_{\mu \nu} & =-\frac{1}{D-2} F^{2} g_{\mu \nu}+\frac{1}{D-2} \Lambda g_{\mu \nu}, \\
R_{m n} & =2 F_{m n}^{2}-\frac{1}{D-2} F^{2} g_{m n}+\frac{1}{D-2} \Lambda g_{m n} \\
& =\frac{D-3}{D-2} F^{2} g_{m n}+\frac{1}{D-2} \Lambda g_{m n}
\end{aligned}
$$

for the spacetime of a direct product. As for the flat $D-2$ dimensional spacetime, where $R_{\mu \nu}=0$, we consider a finetuning of the parameters as

$$
\bar{F}^{2}=\Lambda>0
$$

And, therefore, the solution for the background fields reads

$$
\begin{aligned}
\bar{R} & =2 \bar{F}^{2} \\
\bar{R}_{m n} & =\Lambda \bar{g}_{m n}=2 \bar{F}_{m n}^{2}, \\
\bar{R}_{p q}^{m n} & =\Lambda\left(\delta_{p}^{m} \delta_{q}^{n}-\delta_{q}^{m} \delta_{p}^{n}\right)=2 \bar{F}^{m n} \bar{F}_{p q} .
\end{aligned}
$$

Substituting this compactified background solution into (18), we obtain

$$
\begin{aligned}
-\bar{\nabla}^{2} S^{M N}-\bar{\nabla}^{M} \bar{\nabla}^{N} S+\bar{\nabla}^{M} \bar{\nabla}^{l} S^{L N}+\bar{\nabla}^{N} \bar{\nabla}^{l} S^{L M} \\
-\bar{\nabla}_{P} \bar{\nabla}_{Q} S^{P Q} \bar{g}^{M N}+\bar{\nabla}^{2} S \bar{g}^{M N}+\bar{R}_{L}^{M} S^{L N}+\bar{R}_{L}^{N} S^{L M} \\
+\frac{\alpha}{\beta}\left(S^{M N}-\bar{g}^{M N} S\right)=0 .
\end{aligned}
$$

To show the complete particle spectrum for the ghost field is rather complicated, but we can see that the lowest mass of the ghost is $\sqrt{\alpha / \beta}$ for symmetric tensor fields (which does not couple to the Ricci curvature of the extra space) and vector bosons of the Kaluza-Klein origin (which come from the zero modes of the Lichnerowicz operator on $\left.S^{2},-\bar{\nabla}^{2}+\bar{R}_{m n}\right)$. It is also remarkable that the other graviton and vector field fluctuations are absent in the linearized equation (24).

Thus, taking the analysis by RSS for other fluctuation modes [26] into account, in the classical and linearized analysis, we find that the RSS background solution $M_{D-2} \otimes S^{2}$ is stable if $\alpha / \beta>0$, because there is no growing mode. (Of course, there remains the problem of nonlinear instability and the ghost problem in quantum physics.)

Before closing this section, we note that the effective $D-2$ dimensional Newton constant, which can be found by the coefficient of the Ricci scalar of the $D-2$ dimensional spacetime in the action, is not affected by the higher-order terms in our model and is independent of $\beta$, if the conditions on the background fields (22) and (23) are satisfied. (If we express the $D$-dimensional Ricci scalar as $R=\bar{R}+R_{D-2}$, where $R_{D-2}$ is the Ricci scalar of the $D-2$ dimensional spacetime, the coefficient of $R_{D-2}$ in the Lagrangian $L$ (7) is found to be $\alpha-(1 / 2(D-1)) \beta\left[-D \bar{R}+(D+2) \bar{F}^{2}-(D-2) \Lambda\right]$.) It might be related to the absence of the graviton and the ghost modes in (24) at the linearized level mentioned above.

\section{De Sitter Solutions and Cosmology in the Model}

In this section, we perform the analysis on the stability of the compactification and the cosmological solution in our model. We consider an effective potential $V$ for the radius of extra space $S^{2}$ [27] in order to study them.

We can express the Riemann tensor of the $S^{2}$ as

$$
R_{p q}^{m n}=\frac{1}{b^{2}}\left(\delta_{p}^{m} \delta_{q}^{n}-\delta_{q}^{m} \delta_{p}^{n}\right)
$$

where $b$ denotes the radius of the compactified sphere $S^{2}$. Then, (21) with $R_{\mu \nu}=0$ impose [26]

$$
\begin{aligned}
& q^{2}=\frac{1}{2 \Lambda}, \\
& b^{2}=\frac{1}{\Lambda}
\end{aligned}
$$

where the first equation represents the fine-tuning condition between the parameters. 
The effective potential $V$ as a function of $b$, the scale of the extra space, is obtained by replacing the background fields with functions of $b$ in $-I$ such that

$$
\begin{aligned}
R_{n}^{m} & =\frac{1}{b^{2}} \delta_{n}^{m}, \\
\left(F^{2}\right)_{n}^{m} & =\frac{q^{2}}{b^{4}} \delta_{n}^{m}=\frac{1}{2 \Lambda b^{4}} \delta_{n}^{m},
\end{aligned}
$$

and $\sqrt{\operatorname{det} \bar{g}_{m n}} \propto b^{2}$. The effective potential for the RSS model is calculated from $-I_{0}$ and is given as

$$
V_{0}\left(\Lambda b^{2}\right)=-b^{2}\left(\frac{2}{b^{2}}-\frac{2 q^{2}}{b^{4}}-\Lambda\right)=-2+\frac{1}{\Lambda b^{2}}+\Lambda b^{2} .
$$

Therefore, the effective potential for our model of which the Lagrangian is described by (7) is

$$
\begin{aligned}
& V(y)=\alpha\left[-2+\frac{1}{y}+y\right]+\frac{\beta \Lambda}{4(D-1)}[4(D-2) \\
& \left.-D y+\frac{2 D}{y}-\frac{12(D-2)}{y^{2}}+\frac{7 D-16}{y^{3}}\right] \\
& =\frac{(y-1)^{2}}{4(D-1) y^{3}}[(7 D-16) \beta+2(D-4) \beta y \\
& \left.+(4(D-1) \alpha-D \beta) y^{2}\right],
\end{aligned}
$$

with $y \equiv \Lambda b^{2}$. A minimum of the potential is found at $y=1$ and attains $V(1)=0$. The stability of the radius of the extra sphere is determined by the sign of the second derivative of the potential:

$$
V^{\prime \prime}(1)=2\left(\alpha+2 \frac{D-3}{D-1} \beta \Lambda\right) .
$$

If $D>3$ and $\alpha, \beta>0$, the scale $b=1 / \sqrt{\Lambda}$ is found to be stable. We also find that it is impossible to create another potential minimum satisfying $V=0$ by any fine-tuning of $\beta / \alpha$ in the model.

At a large value of $b, V$ can be indefinitely negative provided that $(D / 4(D-1)) \beta \Lambda-\alpha>0$. In this case, the $D$ dimensional cosmological constant becomes negative. This may not indicate instability of compactification against a large fluctuation, because the higher derivative terms induce a coupling between the compactification scale and the first and second derivatives of an expanding scale factor for the rest of the space.

Therefore, we investigate the cosmological equation including the dynamical scale factor and the compactification scale. The metric is usually assumed as

$$
d s^{2}=-d t^{2}+a^{2}(t) d \Omega_{D-3}^{2}+b^{2}(t) d \Omega_{2}^{2},
$$

where $b(t)$ denotes the radius of the compactified sphere $S^{2}$ and $d \Omega_{2}^{2}$ is the line element in the unit sphere. For the maximally symmetric $(D-3)$ dimensional space, the line element is denoted by $d \Omega_{D-3}^{2}$ here, and its Riemann tensor is normalized to $\widetilde{R}_{k l}^{i j}=k\left(\delta_{k}^{i} \delta_{l}^{j}-\delta_{l}^{i} \delta_{k}^{j}\right)$ with $k=0, \pm 1$, where $i, j, \ldots=1,2, \ldots, D-3$. The symbol $a(t)$ is the scale factor for the $D-3$ dimensional homogeneous and isotropic space.

The Riemann curvature is computed with the metric as

$$
\begin{aligned}
R_{0 j}^{0 i} & =\frac{\ddot{a}}{a} \delta_{j}^{i}, \\
R_{0 n}^{0 m} & =\frac{\ddot{b}}{b} \delta_{n}^{m}, \\
R_{j n}^{i m} & =\frac{\dot{a}}{a} \frac{\dot{b}}{b} \delta_{j}^{i} \delta_{n}^{m}, \\
R_{k l}^{i j} & =\left(\frac{\dot{a}^{2}+k}{a^{2}}\right)\left(\delta_{k}^{i} \delta_{l}^{j}-\delta_{l}^{i} \delta_{k}^{j}\right), \\
R_{p q}^{m n} & =\left(\frac{\dot{b}^{2}+1}{b^{2}}\right)\left(\delta_{p}^{m} \delta_{q}^{n}-\delta_{q}^{m} \delta_{p}^{n}\right),
\end{aligned}
$$

where the $\operatorname{dot}\left({ }^{\circ}\right)$ denotes the derivative with respect to the cosmic time $t$.

Now we can obtain the effective action of the scale factor, $a(t)$, and the compactification scale, $b(t)$, for the RSS model as

$$
\begin{aligned}
I_{0} & \rightarrow \int d t a^{D-3} b^{2}\left[2(D-3) \frac{\ddot{a}}{a}+4 \frac{\ddot{b}}{b}\right. \\
& +4(D-3) \frac{\dot{a}}{a} \frac{\dot{b}}{b}+(D-3)(D-4) \frac{\dot{a}^{2}+k}{a^{2}} \\
& \left.+2 \frac{\dot{b}^{2}+1}{b^{2}}-\frac{1}{\Lambda b^{4}}-\Lambda\right] \\
& \sim \int d t a^{D-3} b^{2}\left[-4(D-3) \frac{\dot{a}}{a} \frac{\dot{b}}{b}\right. \\
& +(D-3)(D-4) \frac{-\dot{a}^{2}+k}{a^{2}}+2 \frac{-\dot{b}^{2}+1}{b^{2}}-\frac{1}{\Lambda b^{4}} \\
& -\Lambda] \equiv \widetilde{I}_{0},
\end{aligned}
$$

where the overall constant which comes from the volume of the space has been omitted. The action written after the symbol “ ” has been integrated by parts.

The effective action for our model can be written, by adopting the auxiliary fields, as

$$
\begin{aligned}
I^{\prime} & \longrightarrow \widetilde{I}_{0} \\
& -\beta \int d t a^{D-3} b^{2}\left\{2 s _ { 0 } \left[-\frac{(D-3)(D-4)}{2} \frac{\dot{a}^{2}+k}{a^{2}}\right.\right. \\
& \left.-2(D-3) \frac{\dot{a}}{a} \frac{\dot{b}}{b}-\frac{\dot{b}^{2}+1}{b^{2}}+\frac{1}{2 \Lambda b^{4}}+\frac{1}{2} \Lambda\right]+2(D
\end{aligned}
$$




$$
\begin{aligned}
& -3) s_{a}\left[-(D-4) \frac{\ddot{a}}{a}-2 \frac{\ddot{b}}{b}-2(D-4) \frac{\dot{a}}{a} \frac{\dot{b}}{b}\right. \\
& -\frac{(D-4)(D-5)}{2} \frac{\dot{a}^{2}+k}{a^{2}}-\frac{\dot{b}^{2}+1}{b^{2}}+\frac{1}{2 \Lambda b^{4}} \\
& \left.+\frac{1}{2} \Lambda\right]+4 s_{b}\left[-(D-3) \frac{\ddot{a}}{a}-\frac{\ddot{b}}{b}-(D-3) \frac{\dot{a}}{a} \frac{\dot{b}}{b}\right. \\
& \left.-\frac{(D-3)(D-4)}{2} \frac{\dot{a}^{2}+k}{a^{2}}-\frac{1}{2 \Lambda b^{4}}+\frac{1}{2} \Lambda\right]-\left[s_{0}^{2}\right. \\
& \left.\left.+(D-3) s_{a}^{2}+2 s_{b}^{2}\right]+\left[s_{0}+(D-3) s_{a}+2 s_{b}\right]^{2}\right\},
\end{aligned}
$$

where we set $S_{0}^{0}=s_{0}, S_{j}^{i}=s_{a} \delta_{j}^{i}$, and $S_{n}^{m}=s_{b} \delta_{n}^{m}$. Here, the equations of motion are omitted, but they can be obtained from the above action. (The Euler equations can be obtained from the action using the command EulerEquations in the add-on Calculus "VariationalMethods" for Mathematica [28].)

We seek for the de Sitter $\left(\otimes S^{2}\right)$ solution, where $k=0$, $a(t)=e^{H_{0} t}$ with constant $H_{0}$, and $b=b_{0}=$ constant are taken as an ansatz. In this case, all the equations of motion become algebraic equations. With the ansatz, the auxiliary variables are expressed as

$$
\begin{aligned}
& s_{0}=s_{a}=\frac{3-2 \Lambda b_{0}^{2}+\left[D(D-3) H_{0}^{2}-\Lambda\right] \Lambda b_{0}^{4}}{2(D-1) \Lambda b_{0}^{4}}, \\
& s_{b} \\
& =\frac{5-2 D+2(D-2) \Lambda b_{0}^{2}-\left[(D-2)(D-3) H_{0}^{2}+\Lambda\right] \Lambda b_{0}^{4}}{2(D-1) \Lambda b_{0}^{4}} .
\end{aligned}
$$

Substituting these auxiliary relations to the other equations, we obtain the following two equations at last:

$$
\begin{aligned}
& -(7 D-16) \beta+12(D-2) \beta \Lambda b_{0}^{2}-2\{2(D-1) \alpha \\
& \left.+\left[(D+2)(D-3)(D-4) H_{0}^{2}+D \Lambda\right] \beta\right\} \Lambda b_{0}^{4} \\
& +4\{2(D-1) \alpha \\
& \left.+\left[D(D-3)(D-4) H_{0}^{2}-(D-2) \Lambda\right] \beta\right\} \Lambda^{2} b_{0}^{6} \\
& +\left\{(D-3)^{2}(D-6)\left(D^{2}-6 D+4\right) H_{0}^{4} \beta\right. \\
& +2(D-3)(D-4) H_{0}^{2}[2(D-1) \alpha-(D-2) \beta \Lambda] \\
& +[-4(D-1) \alpha+D \beta \Lambda] \Lambda\} \Lambda^{2} b_{0}^{8}=0,
\end{aligned}
$$

$$
\begin{aligned}
& 3(7 D-16) \beta-24(D-2) \beta \Lambda b_{0}^{2}+2\{2(D-1) \alpha \\
& \left.+\left[(D+2)(D-2)(D-3) H_{0}^{2}+D \Lambda\right] \beta\right\} \Lambda b_{0}^{4} \\
& +\left\{(D-3)^{2}(D-2)\left(D^{2}-6 D+4\right) H_{0}^{4} \beta\right. \\
& +2(D-2)(D-3) H_{0}^{2}[2(D-1) \alpha-(D-2) \beta \Lambda] \\
& +[-4(D-1) \alpha+D \beta \Lambda] \Lambda\} \Lambda^{2} b_{0}^{8}=0 .
\end{aligned}
$$

The two sets of the solution for $b_{0}$ and $H_{0}$ can be found; one is

$$
\text { (i) } \begin{aligned}
H_{0}^{2} & =0, \\
b_{0}^{2} & =\frac{1}{\Lambda},
\end{aligned}
$$

and the other is

$$
\text { (ii) } \begin{aligned}
H_{0}^{2} & =\frac{D-4}{(D-3)^{3}} \Lambda, \\
b_{0}^{2} & =\frac{D-3}{\Lambda} .
\end{aligned}
$$

These values for solutions are the same as those of the RSS model [29], as expected (if $D=6$ ). In both cases, the values for $s_{0}, s_{a}$, and $s_{b}$ are equal to zero.

Since the simultaneous equations ((36), (37)) are fourthorder algebraic equations, there are two more sets of solutions (in general, the values for $s_{0}, s_{a}$, and $s_{b}$ are not zero for such solutions) at the most. We may infer that for sufficiently small value of $\beta$, only two original solutions ((38), (39)) are real solutions. For $D=6$, only two real solutions ((38), (39)) exist for $\beta \Lambda / \alpha<\approx 2.8$. As a model of the universe, the multiple de Sitter phases would have some significance in cosmology.

We now study the behavior of the small fluctuations around the above exact solutions. In the neighborhood of (i), we set

$$
\begin{aligned}
b(t) & =\frac{1}{\sqrt{\Lambda}}+\Delta b(t), \\
H(t) & =\frac{\dot{a}}{a}=\Delta H(t),
\end{aligned}
$$

$\Delta s_{a}=s_{a}$, and so on. Then, the linearized equations of motion can be expressed as

$$
\begin{aligned}
& (D-3) \Delta s_{a}+2 \Delta s_{b}=0 \\
& (D-1) \Delta \ddot{s}_{a}+\left[2(D-3) \Lambda+(D-1) \frac{\alpha}{\beta}\right] \Delta s_{a}=0 \\
& 2(D-1) \alpha[(D-2) \Delta \ddot{b}+2(D-4) \Lambda \Delta b] \\
& \quad+\frac{D-3}{\sqrt{\Lambda}}[(D-1)(D-2) \alpha+4 \beta \Lambda] \Delta s_{a}=0 \\
& (D-1) \alpha\left[(D-2) \Delta \dot{H}-4 \Lambda^{3 / 2} \Delta b\right] \\
& \quad-[(D-1)(D-2) \alpha-2(D-3) \beta \Lambda] \Delta s_{a}=0 \\
& (D-1) \Delta s_{0}=-2(D-3) \frac{\beta \Lambda}{\alpha} \Delta s_{a} .
\end{aligned}
$$


Note that, for $\beta=0$, the above equations give $\Delta s_{a}=\Delta s_{b}=$ $\Delta s_{0}=0,(D-2) \Delta \ddot{b}+2(D-4) \Lambda \Delta b=0$, and $(D-2) \Delta \dot{H}-$ $4 \Lambda^{3 / 2} \Delta b=0$, which are equivalent to the equations for the cosmology of the RSS model [29].

The oscillatory behavior of $\Delta b$ as well as $\Delta s_{a}$ is already interesting enough. The variables $\Delta s_{0}, \Delta s_{a}$, and $\Delta s_{b}$ oscillate with a different frequency from that of $\Delta b$, as seen from (42) and (43). (Note that the angular frequency of the oscillation of $\Delta s_{0, a, b}$ is the square root of $V^{\prime \prime}(1) / 2 \beta$ in (30).) Thus, the tuning of the initial conditions provides enough time for accelerating expansion and a sufficient inflation can be achieved, though we cannot predict the choice of the initial state in the present classical analysis of the model.

The analysis of the perturbation from (ii) can be performed and it results in finding exponentially growing modes, as found in [29], though we omit the indication of equations here. A certain fine-tuning could give enough expansion time for a sufficient inflation as the de Sitter phase also in this case.

\section{Spontaneous Compactification in Lovelock-Horndeski-Nonlinear Maxwell Theory}

In the solution for the spontaneous compactification in previous sections, one can deduce the following relation in the background fields:

$$
\bar{R}_{M N P Q}=2 \bar{F}_{M N} \bar{F}_{P Q} .
$$

Similar to the consideration in the previous sections, we come up with an idea that the Minkowski compactification of the RSS model is attained in a higher derivative theory if the relation (46) is reflected in the higher-order correction term.

We then propose an additional higher-order term

$$
\begin{aligned}
L_{2}= & \frac{1}{4} \delta_{M N K L}^{P Q R S}\left(R_{P Q}^{M N}-2 F^{M N} F_{P Q}\right)\left(R_{R S}^{K L}-2 F^{K L} F_{R S}\right) \\
= & R^{M N P Q} R_{M N P Q}-4 R^{M N} R_{M N}+R^{2} \\
& -4\left(R^{M N P Q} F_{M N} F_{P Q}-4 R^{M N} F_{M N}^{2}+R F^{2}\right) \\
& +4 F^{2 M N} F_{M N}^{2}-8\left(F^{2}\right)^{2},
\end{aligned}
$$

where generalized Kronecker's delta is defined by

$$
\delta_{N_{1} N_{2} \cdots N_{p}}^{M_{1} M_{2} \cdots M_{p}} \equiv\left|\begin{array}{cccc}
\delta_{N_{1}}^{M_{1}} & \delta_{N_{2}}^{M_{1}} & \cdots & \delta_{N_{p}}^{M_{1}} \\
\delta_{N_{1}}^{M_{2}} & \delta_{N_{2}}^{M_{2}} & \cdots & \delta_{N_{p}}^{M_{2}} \\
\vdots & \vdots & \ddots & \vdots \\
\delta_{N_{1}}^{M_{p}} & \delta_{N_{2}}^{M_{p}} & \cdots & \delta_{N_{p}}^{M_{p}}
\end{array}\right| .
$$

Now, we write model 2 by the Lagrangian (therefore, we call the previous model model 1):

$$
L=\alpha L_{0}+\gamma L_{2} \text {. }
$$

Note that $L_{2}$ consists of the Lanczos-Lovelock Lagrangian (Gauss-Bonnet term) [3-6], the Horndeski's vector-tensor term [18], and a nonlinear Maxwell term.
It is easy to see that adding $L_{2}$ to the RSS Lagrangian $L_{0}$ yields no modification of the linearized equation of motion on the RSS background fields after gauge fixing. Therefore, the Kaluza-Klein modes remain unchanged from those in the RSS model. It is worth noting that the effective $D-2$ dimensional Newton constant in model 2 is not affected by the value of $\gamma$.

Now, we study the cosmological evolution of the classical background in the model. Although the Kaluza-Klein cosmologies with the Gauss-Bonnet term or the generalized Euler invariant have been investigated in many studies including [30-35], the case with the RSS-type compactification with the Gauss-Bonnet term has been studied only in a few papers including [36]. (The compactification with the Horndeski Lagrangian has been considered by one of the present authors [21].)

As previously considered, we characterize the cosmological background by

$$
\begin{aligned}
d s^{2} & =-d t^{2}+a^{2}(t) d \Omega_{D-3}^{2}+b^{2}(t) d \Omega_{2}^{2}, \\
F & =\frac{1}{2} q \varepsilon_{m n} d x^{m} \wedge d x^{n}=\frac{1}{2} \frac{1}{\sqrt{2 \Lambda}} \varepsilon_{m n} d x^{m} \wedge d x^{n} .
\end{aligned}
$$

Then, the Lagrangian $L_{2}$ reduces to

$$
\begin{aligned}
L_{2} & =4(D-3) \frac{\ddot{a}}{a}\left[4(D-4) \frac{\dot{a}}{a} \frac{\dot{b}}{b}\right. \\
& \left.+(D-4)(D-5) \frac{\dot{a}^{2}+k}{a^{2}}+2 \frac{\dot{b}^{2}+1}{b^{2}}-2 \frac{1}{\Lambda b^{4}}\right]+8 \\
& \cdot \frac{\ddot{b}}{b}\left[2(D-3) \frac{\dot{a}}{a} \frac{\dot{b}}{b}\right. \\
& \left.+(D-3)(D-4) \frac{\dot{a}^{2}+k}{a^{2}}\right]+(D-3)(D-4)(D \\
& -5)(D-6)\left(\frac{\dot{a}^{2}+k}{a^{2}}\right)^{2}+8(D-3)(D-4)(D-5) \\
& \cdot \frac{\dot{a}}{a} \frac{\dot{b}}{b} \frac{\dot{a}^{2}+k}{a^{2}}+8(D-3)(D-4) \frac{\dot{a}^{2}}{a^{2}} \frac{\dot{b}^{2}}{b^{2}}+4(D-3) \\
& \cdot(D-4) \frac{\dot{a}^{2}+k}{a^{2}}\left(\frac{\dot{b}^{2}+1}{b^{2}}-\frac{1}{\Lambda b^{4}}\right),
\end{aligned}
$$

and we obtain the following action for $a$ and $b$ :

$$
\begin{aligned}
\widetilde{I}_{2} & =\int d t a^{D-3} b^{2}\{(D-3)(D-4)(D-5)(D-6) \\
\cdot & {\left[-\frac{1}{3} \frac{\dot{a}^{4}}{a^{4}}-2 \frac{k}{a^{2}} \frac{\dot{a}^{2}}{a^{2}}+\frac{k^{2}}{a^{4}}\right]+8(D-3)(D-4) } \\
& \cdot(D-5)\left[-\frac{1}{3} \frac{\dot{a}^{3}}{a^{3}} \frac{\dot{b}}{b}-\frac{k}{a^{2}} \frac{\dot{a}}{a} \frac{\dot{b}}{b}\right]+4(D-3)(D-4)
\end{aligned}
$$




$$
\begin{aligned}
& \cdot\left[-\frac{\dot{a}^{2}}{a^{2}} \frac{\dot{b}^{2}+1}{b^{2}}+\frac{k}{a^{2}} \frac{-\dot{b}^{2}+1}{b^{2}}\right]-4(D-3)(D-4) \\
& \left.\cdot \frac{-\dot{a}^{2}+k}{a^{2}} \frac{1}{\Lambda b^{4}}-16(D-3) \frac{\dot{a}}{a} \frac{\dot{b}}{b} \frac{1}{\Lambda b^{4}}\right\} .
\end{aligned}
$$

Again, we omit the exposition of equations of motion here.

We first explore the de Sitter phase or the maximally symmetric product spacetime. To find this, we set $k=0$, $a(t)=e^{H_{0} t}$, and $b(t)=b_{0}$, where $H_{0}$ and $b_{0}$ are constants. We then obtain the simultaneous algebraic equations as follows:

$$
\begin{aligned}
& -\left[\alpha+4(D-3)(D-4) H_{0}^{2} \gamma\right]+2[\alpha \\
& \left.\quad+2(D-3)(D-4) H_{0}^{2} \gamma\right] \Lambda b_{0}^{2} \\
& \quad+\left[\left((D-3)(D-4) H_{0}^{2}-\Lambda\right) \alpha\right. \\
& \left.\quad+(D-3)(D-4)(D-5)(D-6) H_{0}^{4} \gamma\right] \Lambda b_{0}^{4}=0, \\
& \alpha+4(D-2)(D-3) H_{0}^{2} \gamma \\
& \quad+\left[\left((D-2)(D-3) H_{0}^{2}-\Lambda\right) \alpha\right. \\
& \left.\quad+(D-2)(D-3)(D-4)(D-5) H_{0}^{4} \gamma\right] \Lambda b_{0}^{4}=0 .
\end{aligned}
$$

Of course, the solution (i) (38) is a trivial solution of the above equations, while (ii) (39) is not a solution for $\gamma \neq 0$. One solution of the simultaneous equations ((53), (54)) with $H_{0} \neq$ 0 exists for all positive $\gamma / \alpha$. The value of $b_{0}(>1 / \sqrt{\Lambda})$ increases with the value of $\gamma / \alpha$. The existence of the de Sitter phase with large values for $H_{0}$ and $b_{0}$ is suitable for the possible inflationary phase.

Next, we draw the phase diagram of the compactification scale $b$. We consider the case with $k=0$ and $D=6$. Figure 1 shows the phase space spanned by $b$ and $\dot{b}$ for $\gamma \Lambda / \alpha=0,1,2$. The small arrows in the figures indicate the flow in the phase space. One can see that $b=1 / \sqrt{\Lambda}$ is an attractor and the area of the attracting region increases with the value of $\gamma / \alpha$. (If ordinary matter is added, the dissipative motion becomes more apparent in an expanding era [37].) We conclude that an appropriate choice of the initial condition, which governs the flow passing through the branch point given by the de Sitter solution of (53) and (54), might result in a longer expansion time.

\section{Summary and Prospects}

In this paper, we have considered special cases for extension of the Einstein-Maxwell theory with higher-order terms. The models proposed in the present paper have the same solution for spontaneous compactification $M_{D-2} \otimes S^{2}$ utilizing the internal flux as the RSS model. The stability of the compactified vacuum has been confirmed at the classical level. Nevertheless, the models reveal the different cosmological behaviors because of the additional degrees of freedom or the existence of the additional de Sitter phases. We should investigate these evolutional equations by using numerical calculations in future work.

Our toy models seem to be quite special, but the analysis of the models will exhibit some typical behaviors including inflation, and we can regard general higher-order corrections as a modification to the critical models.

To obtain a realistic inflationary epoch which successfully explain the Planck result on $[38,39]$, we should analyze the models further in detail. Although the possible multiple de Sitter phases and the oscillations around the equilibrium scale certainly lead to an inflationary expansion of the universe in model 1 , it is difficult to specify the initial condition because the dynamical compactification has uncertainty in the beginning of the multidimensional universe. Moreover, initial quantum fluctuations in higher dimensions should be reconsidered to obtain precise values of cosmological perturbations. Due to the same reasons, at the present stage, it is ambiguous whether the possible (tunable) slow-roll inflation in model 2 can explain Planck results. Extensive calculations for various cases are needed to conclude whether our model can completely explain the cosmological observables. (To try to explain the dark energy problem, we will face the finetuning problem in our models, as well as the RSS model.)

Recently, the black holes in higher derivative gravity have attracted renewed interest [40-42]. We consider that our model 1 is appropriate to examine magnetized black holes of a novel type.

The other subjects we should consider are, for instance, the coupling to matter fields; compactifications on $\left(S^{2}\right)^{N}$ [43] in our models; the inclusion of a $p$-form flux field such as the Freund-Rubin compactification [44]; the treatment of our models in quantum cosmology [45-47]; the initial singularity problems [48] in the models; the possible supersymmetrization of the models; and the field content of the model in $3+2$ dimensions.

The generalization of our model 1 to higher-order theory is considered a straightforward exercise. If we introduce an auxiliary field, $S_{M}^{N}$, the additional term can be replaced by

$$
\begin{aligned}
& n \delta_{N_{1} \cdots N_{n-1} N}^{M_{1} \cdots M_{n-1} M} S_{M_{1}}^{N_{1}} \cdots S_{M_{n-1}}^{N_{n-1}}\left(\Delta_{M}^{N}{ }^{P Q} T_{P Q}-S_{M}^{N}\right) \\
& \quad+\delta_{N_{1} \cdots N_{n}}^{M_{1} \cdots M_{n}} S_{M_{1}}^{N_{1}} \cdots S_{M_{n}}^{N_{n}},
\end{aligned}
$$

which will be studied extensively elsewhere.

Finally, we must research the relation of our work to other theories. We suppose that the structure of model 1 owes itself to the matrix $\Delta_{M N P Q}$, which appears also in the spintwo propagator. It would be very interesting if our models 


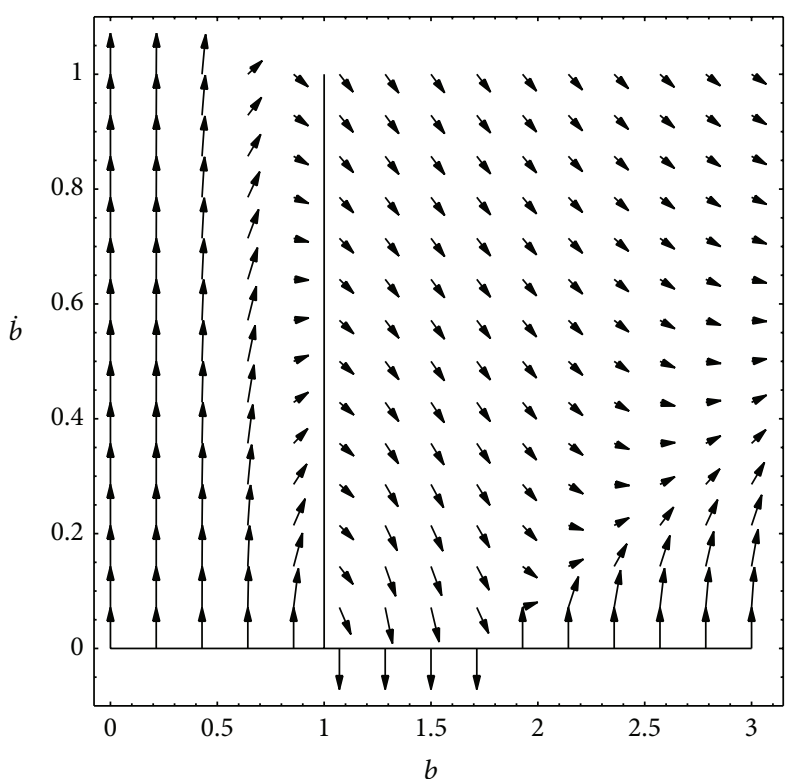

(a)

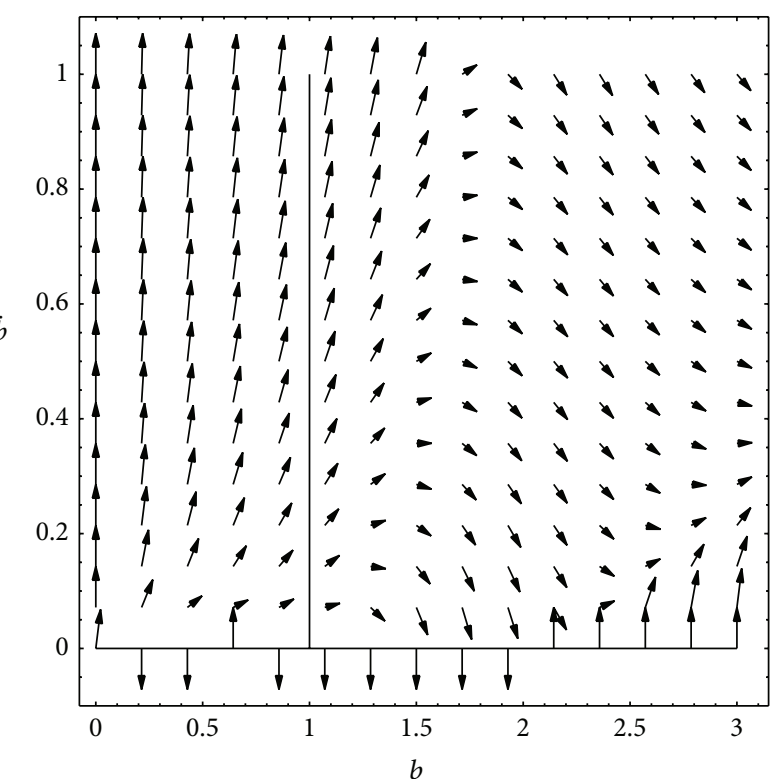

(b)

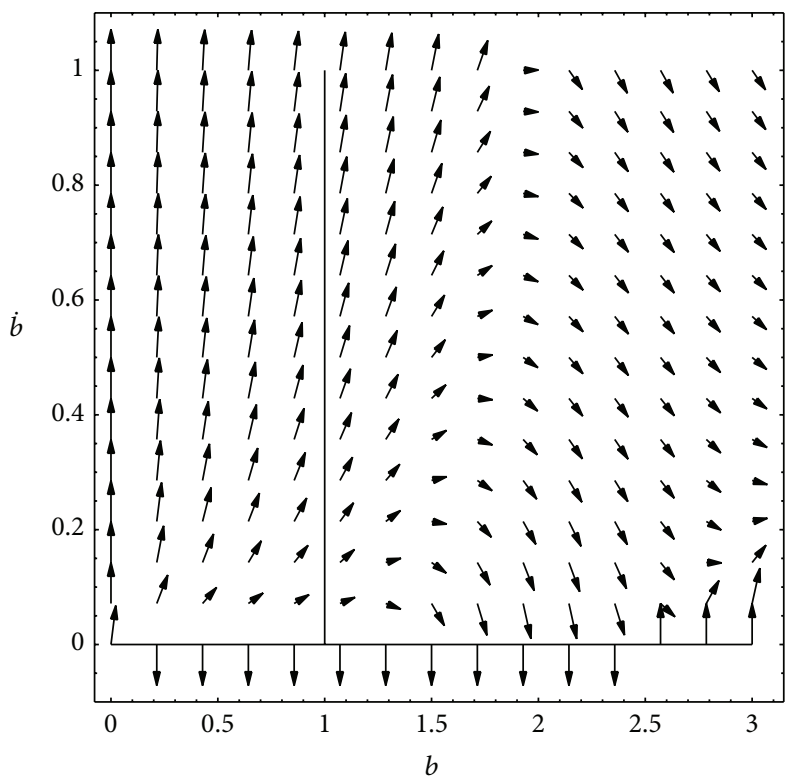

(c)

Figure 1: Phase space for model 2. We consider here a unit such that $\Lambda=1$. We show (a) for $\gamma / \alpha=0$, (b) for $\gamma / \alpha=1$, and (c) for $\gamma / \alpha=2$, respectively.

could be regarded as an effective reformulation or revision of another theory.

\section{Conflict of Interests}

The authors declare that there is no conflict of interests regarding the publication of this paper.

\section{References}

[1] K. S. Stelle, "Renormalization of higher-derivative quantum gravity," Physical Review D, vol. 16, no. 4, pp. 953-969, 1977.
[2] K. S. Stelle, "Classical gravity with higher derivatives," General Relativity and Gravitation, vol. 9, no. 4, pp. 353-371, 1978.

[3] C. Lanczos, "Elektromagnetismus als natürliche Eigenschaft der Riemannschen Geometrie," Zeitschrift für Physik, vol. 73, no. 34, pp. 147-168, 1932.

[4] C. Lanczos, "A remarkable property of the Riemann-Christoffel tensor in four dimensions," The Annals of Mathematics, vol. 39, no. 4, pp. 842-850, 1938.

[5] D. Lovelock, "The Einstein tensor and its generalizations," Journal of Mathematical Physics, vol. 12, no. 3, article 498, 1971.

[6] D. Lovelock, "The four-dimensionality of space and the Einstein tensor," Journal of Mathematical Physics, vol. 13, pp. 874-876, 1972. 
[7] T. Padmanabhan and D. Kothawala, "Lanczos-Lovelock models of gravity," Physics Reports, vol. 531, no. 3, pp. 115-171, 2013.

[8] B. Zumino, "Gravity theories in more than four dimensions," Physics Reports, vol. 137, no. 1, pp. 109-114, 1986.

[9] B. Zwiebach, "Curvature squared terms and string theories," Physics Letters B, vol. 156, no. 5-6, pp. 315-317, 1985.

[10] H. Lü and C. N. Pope, "Critical gravity in four dimensions," Physical Review Letters, vol. 106, no. 18, Article ID 181302, 2011.

[11] S. Deser, H. Liu, H. Lü, C. N. Pope, T. Ç. Şişman, and B. Tekin, "Critical points of $D$-dimensional extended gravities," Physical Review D, vol. 83, no. 6, Article ID 061502(R), 2011.

[12] H. Lü, Y. Pang, and C. N. Pope, "Conformal gravity and extensions of critical gravity," Physical Review D, vol. 84, no. 6, Article ID 064001, 9 pages, 2011.

[13] M. Alishahiha and R. Fareghbal, " $D$-dimensional log gravity," Physical Review D, vol. 83, no. 8, Article ID 084052, 2011.

[14] E. A. Bergshoeff, O. Hohm, J. Rosseel, and P. K. Townsend, "Modes of log gravity," Physical Review D, vol. 83, no. 10, Article ID 104038, 2011.

[15] N. Johansson, A. Naseh, and T. Zojer, "Holographic two-point functions for 4d log-gravity," Journal of High Energy Physics, vol. 2012, no. 9, article 114, 2012.

[16] E. A. Bergshoeff, O. Hohm, and P. K. Townsend, "Massive gravity in three dimensions," Physical Review Letters, vol. 102, no. 20, Article ID 201301, 2009.

[17] E. A. Bergshoeff, O. Hohm, and P. K. Townsend, "More on massive 3D gravity," Physical Review D, vol. 79, no. 12, Article ID 124042, 2009.

[18] G. W. Horndeski, "Conservation of charge and the EinsteinMaxwell field equations," Journal of Mathematical Physics, vol. 17, no. 11, pp. 1980-1987, 1976.

[19] J. D. Barrow, M. Thorsrud, and K. Yamamoto, "Cosmologies in Horndeski's second-order vector-tensor theory," Journal of High Energy Physics, vol. 2013, no. 2, article 146, 2013.

[20] J. Beltrán Jiménez, R. Durrer, L. Heisenberg, and M. Thorsrud, "Stability of Horndeski vector-tensor interactions," Journal of Cosmology and Astroparticle Physics, vol. 2013, no. 10, article 064, 2013.

[21] K. Yoshida and K. Shiraishi, "A new vector-tensor theory and higher-dimensional cosmology," Physica Scripta, vol. 43, no. 2, pp. 129-132, 1991.

[22] N. Kan, K. Kobayashi, and K. Shiraishi, "'Critical' cosmology in higher order gravity," ISRN Mathematical Physics, vol. 2013, Article ID 651684, 9 pages, 2013.

[23] N. Kan, K. Kobayashi, and K. Shiraishi, "Critical higher order gravities in higher dimensions," Physical Review D, vol. 88, no. 4, Article ID 044035, 2013.

[24] N. H. Barth and S. M. Christensen, "Quantizing fourth-order gravity theories: the functional integral," Physical Review D, vol. 28, no. 8, pp. 1876-1893, 1983.

[25] M. Fierz and W. Pauli, "On relativistic wave equations for particles of arbitrary spin in an electromagnetic field," Proceedings of the Royal Society of London Series A: Mathematical and Physical Sciences, vol. 173, no. 953, pp. 211-232, 1939.

[26] S. Randjbar-Daemi, A. Salam, and J. Strathdee, "Spontaneous compactification in six-dimensional Einstein-Maxwell theory," Nuclear Physics B, vol. 214, no. 3, pp. 491-512, 1983.

[27] C. Wetterich, "Spontaneous compactification in higher dimensional gravity," Physics Letters B, vol. 113, no. 5, pp. 377-381, 1982.

[28] Wolfram, http://www.wolfram.com/.
[29] Y. Okada, "Inflation in Kaluza-Klein cosmology," Physics Letters $B$, vol. 150, no. 1-3, pp. 103-106, 1985.

[30] J. Madore, "Kaluza-Klein theory with the Lanczos lagrangian," Physics Letters. A, vol. 110, no. 6, pp. 289-292, 1985.

[31] F. Müller-Hoissen, "Dimensionally continued Euler forms: Kaluza-Klein cosmology and dimensional reduction," Classical and Quantum Gravity, vol. 3, no. 4, p. 665, 1986.

[32] A. B. Henriques, "Higher-dimensional cosmological solutions with generalized Einstein equations," Nuclear Physics B, vol. 277, pp. 621-633, 1986.

[33] K. Shiraishi, "The Friedmann universe and compact internal spaces in higher-dimensional gravity theories," Progress of Theoretical Physics, vol. 76, no. 1, pp. 321-324, 1986.

[34] M. Arik, "The analogue potential of Kaluza-Klein cosmology with Euler form actions," Nuclear Physics B, vol. 328, no. 1, pp. 308-316, 1989.

[35] N. Deruelle and L. Fariña-Busto, "Lovelock gravitational field equations in cosmology," Physical Review D, vol. 41, no. 12, pp. 3696-3708, 1990.

[36] S. Mignemi, "Spontaneous compactification in 6-dimensional einstein-lanczos-maxwell theory," Modern Physics Letters A, vol. 1, no. 5, pp. 337-343, 1986.

[37] K. Maeda, "Stability and attractor in a higher-dimensional cosmology. I," Classical and Quantum Gravity, vol. 3, no. 2, pp. 233-247, 1986.

[38] P. A. R. Ade, N. Aghanim, M. Arnaud et al., "Planck 2015 results. XIII. Cosmologicalparameters," http://arxiv.org/abs/1502.01589.

[39] Planck Collaboration, "Planck 2015 results. XX. Constraints on inflation," http://arxiv.org/abs/1502.02114.

[40] H. Lü, A. Perkins, C. N. Pope, and K. S. Stelle, "Black holes in higher derivative gravity," Physical Review Letters, vol. 114, no. 17, Article ID 171601, 2015.

[41] H. Lü, A. Perkins, C. N. Pope, and K. S. Stelle, "Black holes in $D=4$ higher-derivative gravity," International Journal of Modern Physics A, vol. 30, Article ID 1545016, 2015.

[42] A. Kehagias, C. Kounnas, D. Lüst, and A. Riotto, "Black hole solutions in $R^{2}$ gravity," Journal of High Energy Physics, vol. 2015, no. 5, article 143, 2015.

[43] A. R. Brown, A. Dahlen, and A. Masoumi, "Flux compactifications on $\left(S_{2}\right)^{N}$," Physical Review D, vol. 90, no. 4, Article ID 045016, 2014.

[44] P. G. O. Freund and M. A. Rubin, "Dynamics of dimensional reduction," Physics Letters B, vol. 97, no. 2, pp. 233-235, 1980.

[45] J. J. Halliwell, "The quantum cosmology of Einstein-Maxwell theory in six dimensions," Nuclear Physics B, vol. 266, no. 1, pp. 228-244, 1986.

[46] Y. Zhong and X.-Z. Li, "Quantum cosmology of Einstein-KalbRamond theory," Physical Review D, vol. 42, no. 2, pp. 712-715, 1990.

[47] Y. Zhong and X.-Z. Li, "The quantum cosmology of 4-form fields in $d=11$ spacetime," Communications in Theoretical Physics, vol. 15, no. 1, pp. 113-118, 1991.

[48] A. A. Starobinsky, "A new type of isotropic cosmological models without singularity," Physics Letters B, vol. 91, no. 1, pp. 99-102, 1980. 

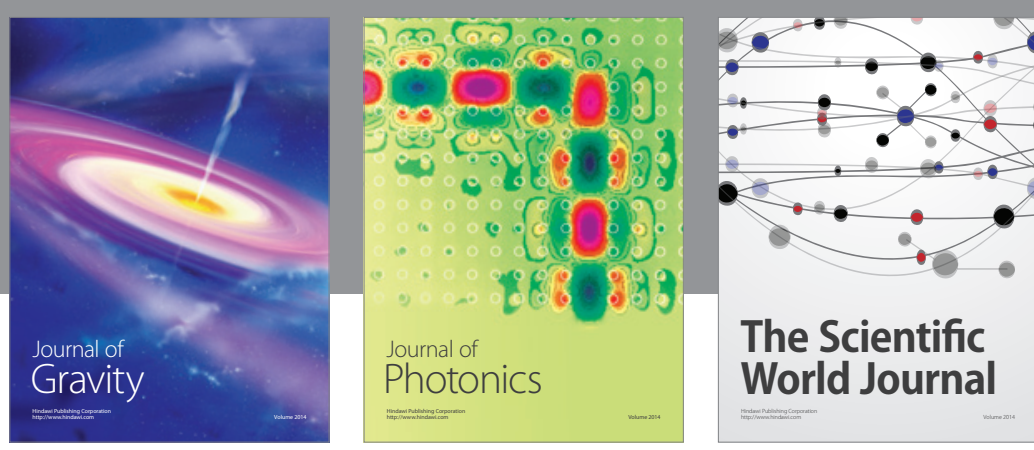

The Scientific World Journal
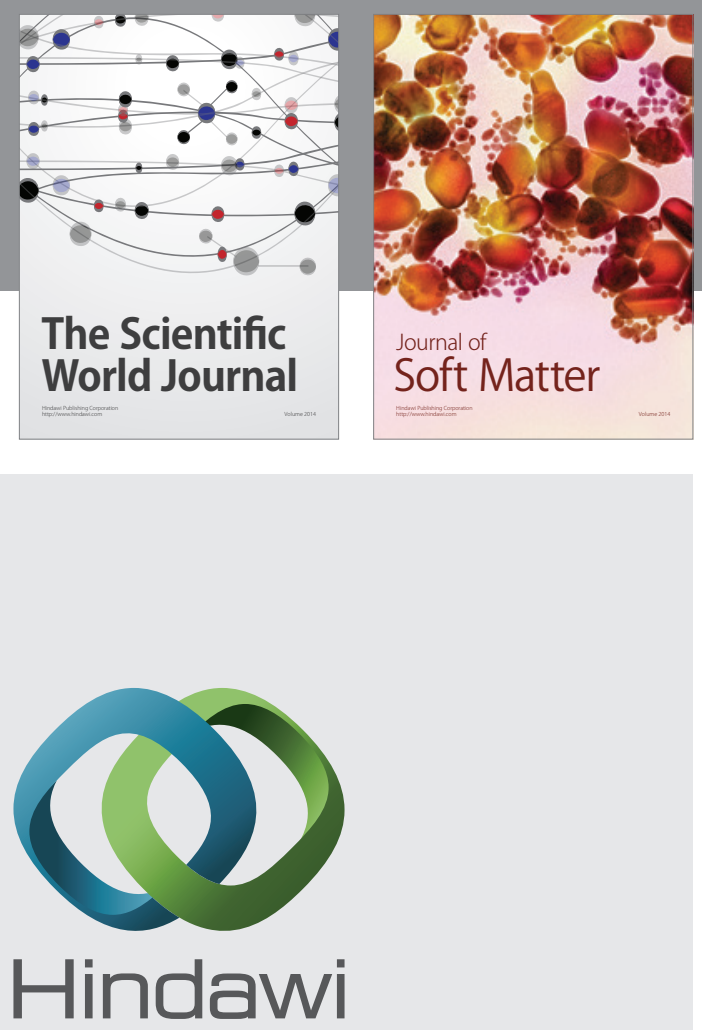

Submit your manuscripts at

http://www.hindawi.com

nternational Journal of

Statistical Mechanics
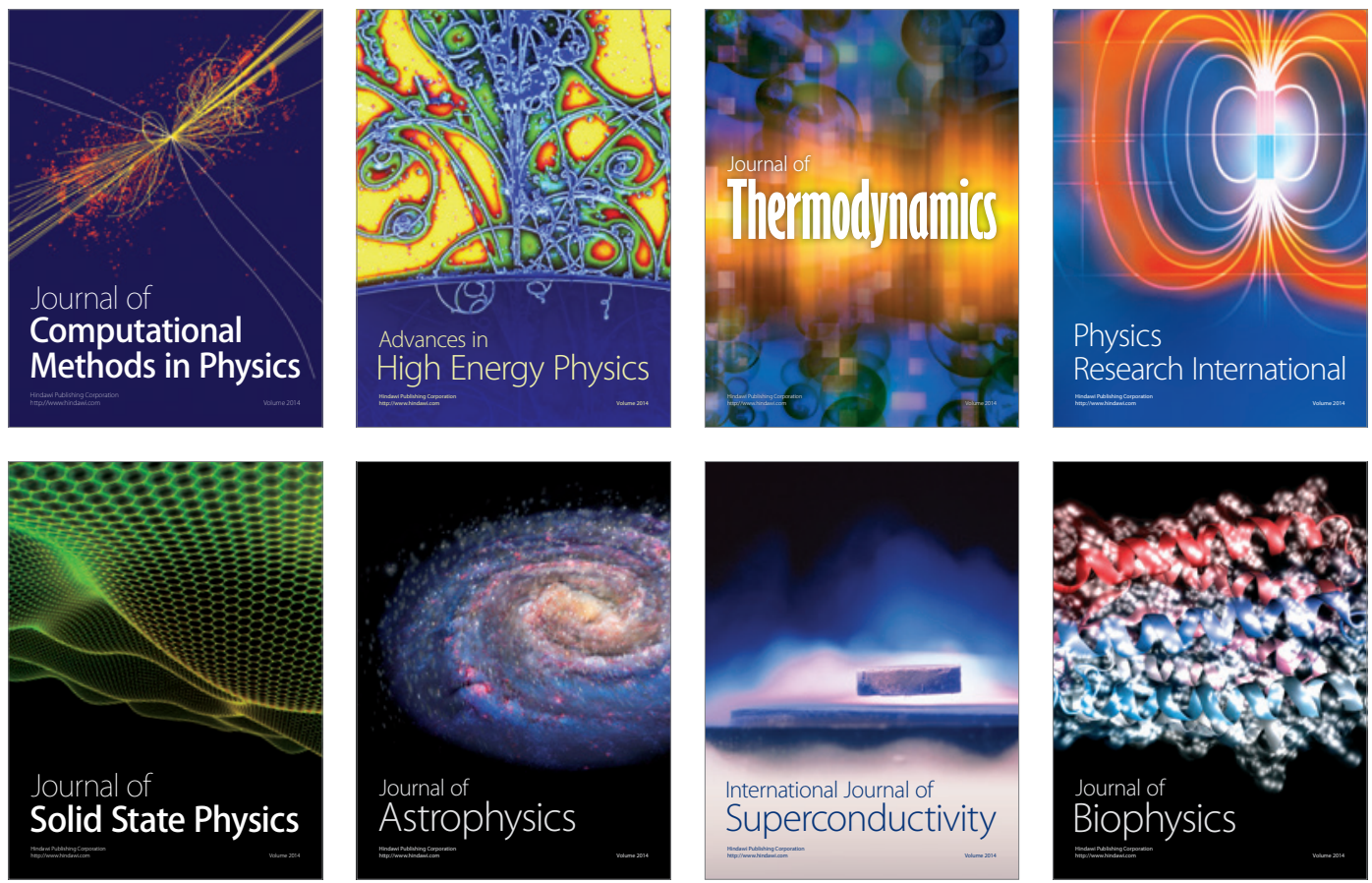
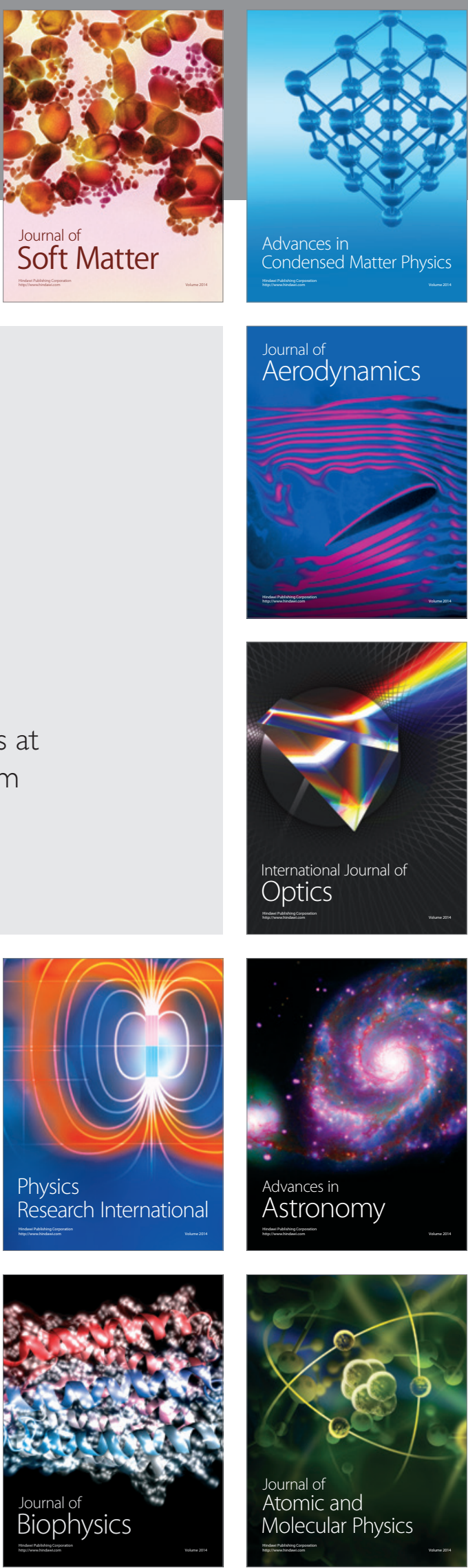